\title{
Die Beziehung zwischen Familienrecht und Human-/ Sozialwissenschaften am Beispiel des Kindschaftsrechts
}

\section{The relationship between family law and the humanities/social sciences exemplified by the law of parent and child}

\section{Zusammenfassung}

Die Beziehung zwischen Kindschaftsrecht und den Human-/Sozialwissenschaften ist so offensichtlich, dass eine Klärung und Systematisierung dieses Verhältnisses überflüssig schien. Der Ruf nach einer Integration der Human- und Sozialwissenschaften in Rechtswissenschaft und in Rechtsanwendung war in den 1970er und 1980er Jahren des vergangenen Jahrhunderts en vogue; sie wurde in Ansätzen verwirklicht, aber auch wieder zurückgenommen. Die Notwendigkeit, Erkenntnisse der Human-/Sozialwissenschaften in der Familienrechtswissenschaft, der Gesetzgebung und der Rechtspraxis zu beachten, ist aktueller denn je. In dieser Abhandlung können lediglich die Berührungsbereiche am Beispiel des Kindschaftsrechts aufgezeigt werden. Für das Kindschaftsrecht besteht die berechtigte Hoffnung, dass Gesetzgebung und Rechtsanwendung unter Heranziehung und Einbeziehung human-/sozialwissenschaftlicher Methoden und Wissensbestände noch am ehesten eine dem Wohl des Kindes am besten gerecht werdende - besser: eine dem Wohl des Kindes am wenigsten schädliche - Alternative finden.

Schlüsselwörter: Kindschaftsrecht, Integration der Human- und Sozialwissenschaften, Rechtsanwendung, Familienrechtswissenschaft

\begin{abstract}
The relationship between the law of parent and child and the humanities/social sciences seems to be so obvious that a clarification and systematization of this relationship is regarded as unnecessary. The call for an integration of the humanities and the social sciences in legal studies and legal practice was very pronounced during the $1980 \mathrm{~s}$ and 1990s. This integration has been partially realized back then, but was revoked later on. The need for taking findings from the humanities and social sciences in law studies, legislation, and legal practice into account is more relevant now than it was ever before. In the present treatise, only the disciplines' interwoven areas pertaining to the law of parent and child can be identified. Regarding the law of parent and child, there is reasonable hope that legislation and legal practice by means of drawing on and integrating humanities' and social sciences' methods and knowledge bases may be able to find alternatives that meet the best interest of the child (or at least constitute the least detrimental alternative).
\end{abstract}

Key words: law of parent and child, integration of the humanities and social sciences, legal practice, family law studies 


\section{Einleitung}

Die Existenz einer solchen Beziehung lässt sich wohl kaum bestreiten. Bekanntlich gibt es gute und weniger gute Beziehungen, auch heimliche Beziehungen, aber auch weniger gern gepflegte, solche, deren Existenz man sich nicht bewusst ist oder von denen man nichts wissen will. Sicherlich handelt sich bei dieser Beziehung bislang nicht um eine geregelte, systematisch durchdachte oder strukturierte. Unter diesen Umständen bleibt es den ,individuellen Neigungen und Erkenntnisinteressen“ (Röthel 2015: 192) einzelner Familierechtswissenschaftler, der Rechtspolitik und nicht zuletzt dem Bundesverfassungsgericht (BVerfG) überlassen, ob, wie und in welchem Ausmaß „Nachbarwissenschaften“ für das Familien-, insbesondere für das Kindschaftsrecht Bedeutung erlangen. Die Familienrechtswissenschaft hat es bislang nicht geschafft diese zweifellos vorhandenen und notwendigen Beziehungen zu klären. Was die unleugbare „besondere Nähe [des Familienrecht] zu den Sozial- und Lebenswissenschaften“" (idb.: 189) konkret bedeutet, bleibt im Ungefähren. Dies mag auch mit dem noch keineswegs abgeschlossenen Prozess der Selbstvergewisserung und Selbstbehauptung der Familienrechtswissenschaft zusammenhängen, hat sie doch „keinen leichten Stand innerhalb der Rechtswissenschaft“ (ibd.: 183). Und selbst unter „Familienrechtlern“ gelten solche Kolleg(inn)en, die sich mit Kindschaftsrecht und Jugendhilferecht beschäftigen, als bedauernswerte Exoten. Diejenigen, die auch noch eine „voraussetzungsvolle Öffnung“ des Familienrechts verlangen, sind „Außenseiter" geblieben (ibd.: 191), ist doch vor unkontrollierten „Unterspülungen“ durch die Nachbarwissenschaften gewarnt worden. Da helfen nur noch Betretungsverbote, Aufschüttungen, Deiche, Seile, Pflöcke, Rettungsbote oder -ringe, das Heil wird in einer Redogmatisierung (Simitis 1994: 445) des Familienrechts gesucht, um als eine ordentliche Disziplin innerhalb der Rechtswissenschaft endlich Anerkennung zu finden. Aber eine „heile “ - nur auf das reine Recht beschränkte - Welt wird es nicht mehr geben. Interdisziplinarität ist nun einmal anstrengend und voraussetzungsvoll, dennoch unausweichlich.

\subsection{Themen des Kindschaftsrechts}

Bereits ein Blick auf die Themen der Verfahren in Kindschaftssachen ( $§ 151$ FamFG) lässt sofort erkennen, dass sich zahlreiche human-/sozialwissenschaftliche Bezüge geradezu aufdrängen:

„Kindschaftssachen sind die dem Familiengericht zugewiesenen Verfahren, die

1. die elterliche Sorge,

2. das Umgangsrecht und das Recht auf Auskunft über die persönlichen Verhältnisse des Kindes,

3. die Kindesherausgabe,

4. die Vormundschaft,

5. die Pflegschaft oder die gerichtliche Bestellung eines sonstigen Vertreters für einen Minderjährigen oder für eine Leibesfrucht,

6. die Genehmigung der freiheitsentziehenden Unterbringung eines Minderjährigen ( $\S$ 1631b, 1800 und 1915 des Bürgerlichen Gesetzbuchs), 
7. die Anordnung der freiheitsentziehenden Unterbringung eines Minderjährigen nach den Landesgesetzen über die Unterbringung psychisch Kranker oder

8. (..)

betreffen".

\subsection{Bedeutung der Nachbarwissenschaften}

Aus dem Fächerkanon der Human-/Sozialwissenschaften haben insbesondere Psychologie, Erziehungswissenschaften, Medizin, Psychiatrie, Soziologie, Verhaltensbiologie und Sozialarbeit/-pädagogik für das Familien- und vor allem für das Kindschaftsrecht hervorragende Bedeutung. Das materielle Kindschaftsrecht und die es betreffenden gerichtlichen und behördlichen Verfahren kommen heutzutage kaum mehr ohne Befunde der sozialund humanwissenschaftlichen Forschung und Praxis aus. Das gilt sowohl bereits für Familienrechtswissenschaft und Reformgesetzgebung in diesem Bereich - hier müssen sich Grundannahmen, Notwendigkeit und Stoßrichtung human-/sozialwissenschaftlich vergewissern - als auch für die Rechtsanwendung - hier ist die gerichtliche und behördliche Praxis häufig auf Zuarbeit aus den Humanwissenschaften angewiesen, ohne dass die Letztentscheidungzuständigkeit und -verantwortung abgegeben werden darf. Gesetzgeber bleibt Gesetzgeber und der Richter bleibt Richter, auch wenn sozial- und humanwissenschaftliche Befunde bei ihrer jeweils spezifischen Entscheidungssituation Berücksichtigung finden. „Ein ernst genommener interdisziplinärer Diskurs (entläßt) die Juristen eben nicht aus der eigenen Verantwortung, sondern (verlangt) von ihnen, ihr Instrumentarium kritisch mit Hilfe der in den anderen, außerjuristischen Disziplinen entwickelten Ansätze zu überprüfen, ohne sich jemals blind auf irgendeinen dieser Ansätze zu verlassen“ (Simitis 1994: 439).

\section{Evaluation, Rechtstatsachenforschung, Rechtspolitik, wissenschaftliche Politikberatung}

In einer zunehmenden Anzahl von Ländern erlangen Implementations- und Rechtstatsachenforschung eine wachsende Bedeutung, wollen doch Gesetzgeber ihre Intentionen verwirklicht sehen und erforderlichenfalls nachbessern. Realitätsmessung und Wirkungsforschung sind ohne sozialwissenschaftliche Unterstützung nicht möglich. Das ist weit mehr als die beklagte „zunehmende Zahlenorientierung der Familienrechtswissenschaft“ (Röthel 2015: 189). In Ländern des anglo-amerikanischen Rechtskreises sind so genannte socio-legal studies und eigens darauf ausgerichtete Forschungszentren seit Jahrzehnten etabliert, deren sich die Familienrechtswissenschaft und die Rechtspolitik im Rahmen von Implementationsforschung und für Evaluationen von Regelungssystemen laufend bedient. Immerhin verabschiedet auch der bundesrepublikanische Gesetzgeber in jüngster Zeit zunehmend mehr Gesetze mit so genannten Evaluationsklauseln (Z.B. Art. 4 Bundeskinderschutzgesetz (hierzu s. Bundestags-Drucks. 18/7100, Bericht über die Evaluation des Bundeskinderschutzgesetzes) und Artikel 6 Gesetz zur Reform der elterlichen Sorge nicht 
miteinander verheirateter Eltern), indes handelt es sich hier immer noch eher um Ausnahmen und nicht um eine etablierte Praxis. Welche Ergebnisse die neuen Evaluationsklauseln bringen, von welchem Nutzen sie für Familienrechtswissenschaft, Rechtspolitik und die Praxis sein werden, lässt sich noch nicht absehen. Es ist aber auf jeden Fall ein positives Zeichen, dass der Gesetzgeber selbst mit Fristsetzungen versehenen Überprüfungsmechanismen die Anwendung und die Wirkungen seiner Gesetze kontrollieren will; zudem haben solche vom Bundestag in Gesetzesform gegossene Evaluationsklauseln den Vorteil, dass die Evaluation nicht auf die lange Bank geschoben werden kann (wie es z.B. hinsichtlich der Überprüfungen der Wirkungen des Gesetzes zur Reform des Verfahrens in Familiensachen und in den Angelegenheiten der freiwilligen Gerichtsbarkeit (FGG-RG (2009)) der Fall war; hier bestanden zunächst nur Absichtserklärungen, fünf Jahre nach Inkrafttreten Untersuchungen auf den Weg zu bringen. Evaluationsklauseln in Gesetzen oder Koalitionsvereinbarungen haben den Vorteil, dass zugleich die dafür erforderlichen Haushaltsmittel zur Verfügung gestellt werden müssen. Die Abteilung Rechtstatsachenforschung im Bundesministerium der Justiz und für Verbraucherschutz (BMJV) scheint im Vergleich zu den 1980er und 1990er Jahren des vergangenen Jahrhunderts bedauerlicherweise an Bedeutung verloren zu haben. Bemerkenswert in diesem Zusammenhang ist, dass z.B. im aktuellen Koalitionsvertrag der Regierungsparteien sich nachfolgende Passage findet: „Wir wollen das Ineinandergreifen von Gewaltschutz und Umgangsrecht in Bezug auf das Kindeswohl wissenschaftlich untersuchen" (Koalitionsvertrag 2013: 70). Inzwischen ist auch dieser Untersuchungsauftrag vergeben.

Dass sich zentrale und dringend stellende Fragen des Kindschaftsrechts mit einer ausgefeilten Rechtsdogmatik oder - um beim Beispiel des Umgangsrechts zu bleiben - mit einem ausgeklügelten System des Verfahrens-, insbesondere des Vollstreckungsrechts lösen ließen, konnte ernsthaft kaum erwartet werden. Zwischen 1999 (27.754 Verfahren) und 2013 (56.410 Verfahren) haben sich die gerichtlichen Regelungsverfahren des Umgangs mehr als verdoppelt. Bis heute liegt keine Untersuchung vor, die diesen Anstieg und die dahinter stehende Konfliktdynamik zu klären versucht. Hätten sich der Gesetzgeber des Kindschaftsrechtsreformgesetzes (KindRG; Bundesgesetzblatt (BGB1). 1997 I: 2942) und des Gesetzes zur Reform des Verfahrens in Familiensachen und in Angelegenheiten der freiwilligen Gerichtsbarkeit (FGG-RG; BGBl. 2008 I, S. 2596) rechtzeitig, intensiver und offener human- und sozialwissenschaftlicher Rückversicherung bedient und auch rechtsvergleichend gearbeitet, so hätten manche Schwierigkeit, Frustration, Enttäuschung, Belastung oder gar Gefährdung des Kindeswohls im Umgangskontext vermieden werden und überzogene Erwartungen gedämpft können.

\section{Interdisziplinarität als Schutz vor, groben Daumenregeln““}

Schnell und ohne großen Widerstand - trotz innerer Vorbehalte und trotz Missbehagens bei etlichen, die aber aus Gründen der political correctness nicht offen ausgesprochen werden - fällt der Ruf nach Interdisziplinarität, hierfür erforderliche Zeit- und materielle Ressourcen werden häufig nicht mitbedacht. Allerdings werden Familienrechtler schnell merken, dass dieser Weg anstrengend ist, zudem die herbeigerufenen Human- und Sozi- 
alwissenschaften auch nicht frei von Widersprüchen und Aporien sind (Simitis 1994: 425ff.), absolute Gewissheiten wird hoffentlich niemand erwarten. Außerdem stehen Konfliktkonstellationen und Entscheidungen (vgl. ibd.: 440), wie sie nun einmal von Familiengerichten und Sozialbehörden zu treffen sind, nicht im ständigen Fokus der relevanten human-/sozialwissenschaftlichen Forschung, so dass einschlägige Erkenntnisse bei Bedarf dort jederzeit abgerufen werden könnten, vielmehr müssen aufwändige Transfer(Hartwig/Rebe 1978: 24ff) und Übersetzungsleistungen im Hinblick auf die relevanten Themen und Fragen unter penibler Berücksichtigung der juristischen Relevanzkriterien und Verfahren erbracht werden. Der anspruchsvolle interdisziplinäre Weg scheint dennoch noch am geeignetsten und erfolgversprechendsten, um Irrtümern zu entgehen, um sich nicht von tagespolitischen Ereignissen oder von ideologiegeleiteten, medial erregten Kampagnen überwältigen zu lassen. Immer wieder wird die Praxis, aber auch die Rechtspolitik mit Ideologien oder empirisch nicht haltbaren „Theorien“ überrannt, wobei selbst vor Manipulationen von eindeutigen empirisch gesicherten Ergebnissen keine Scheu besteht. Die Bespiele sind zahlreich:

- Das „Parental Alienation Syndrom“ (PAS) sei ein überragendes Erklärungs- und Lösungsmodell bei Umgang verweigernden Kindern (kritisch hierzu Fegert, ZJK 2013: 190).

- Das „Cochemer Modell“ mache gerichtliche Entscheidungen überflüssig (hierzu Salgo 2009: 155).

- Ein Kind bräuchte immer zwei Eltern.

- Ein gesundes Kind hielte Bindungs- und Beziehungsabbrüche, mehrfache „Verpflanzungen" ohne langfristige Folgen aus (missverständlich und ohne empirische Nachweise: Kindler 2013: 194, 199).

- Umgang sei immer wichtig.

- Biologische Eltern seien immer die besten.

- Jede Familie sei besser als ein Heim.

- Nicht bei ihren biologischen Eltern aufwachsende Kinder hätten immer „Heimweh“ nach diesen.

- Schläge hätten noch keinem geschadet.

- Das Miterleben von ,häuslicher Gewalt" schade Kindern nicht.

- Ein „Doppelresidenzmodell“ bei getrennt lebenden Eltern sei immer - auch und gerade bei „Hochstrittigkeit“ - die beste Lösung (hierzu kritisch Kostka 2014).

Die meisten dieser beispielhaft ausgewählten Annahmen und Alltagstheorien kollidieren bereits mit dem geltenden Recht, zudem sind sie längst wissenschaftlich widerlegt. Dennoch leben solche ,groben Daumenregeln“ (Coester 1983: 178) in der Praxis lange fort oder wieder auf. Unentschiedenheit und Uneindeutigkeit der Gesetzgebung sowie der unvermeidliche Einsatz von unbestimmten Rechtsbegriffen und Generalklauseln im Kindschaftsrecht (Simitis 1986: 605f) befördern geradezu das Fortleben von Aberglauben. 


\section{4. „Legitimation durch Verfahren“}

Der Gesetzgeber hat solche Fallstricke durchaus erkannt und „zähmt“ die nur scheinbar unbeschränkte Macht des Familiengerichts durch eine Reihe von zwingenden Verfahrensregelungen („Legitimation durch Verfahren“ (Luhmann 1983), die einerseits dem Rechtsstaatsgebot und der Grundrechtsbindung zu verdanken sind, andererseits gezielt den Einfluss außerrechtlicher Wissensbestände in und auf den „Rechtsfall“ zu sichern suchen. Ein Verzicht auf die Ausschöpfung bzw. den Einsatz dieser Erkenntnisquellen bzw. Verfahrensschritte macht die Entscheidung fehlerbehaftet und wird mit nicht geringer Wahrscheinlichkeit zur Aufhebung der Entscheidung durch das OLG führen, sofern dies von einem hierzu Berechtigten im Beschwerdeverfahren geltend gemacht wird:

- Amtsermittlungsgrundsatz (§ 26 FamFG (Gesetz über das Verfahren in Familiensachen und in den Angelegenheiten der freiwilligen Gerichtsbarkeit))

- Elternanhörung ( $\$ 160$ FamFG)

- Kindesanhörung ( $\$ 159$ FamFG)

- Interessenvertretung durch einen Verfahrensbeistand für das Kind (§ 158 FamFG)

- Mitwirkung des Jugendamtes (§162 FamFG)

- Mitwirkung der Pflegeperson (§161 FamFG)

- Gutachten (§163 FamFG)

- Herstellung von Einvernehmen bei Elternstreit ( $\$ 156$ FamFG)

- Erörterungsgespräch zur Steigerung der Hilfeakzeptanz bei Kindeswohlgefährdung ( $\S$ 157 FamFG; s. Berneiser 2015)

"Gute professionelle Arbeit verlangt das Gewichten, Abwägen und Auflösen widersprüchlicher Anforderungen (...) zwischen fachlich begründeten Überzeugungen und persönlichen Wertvorstellungen (...), damit nicht im Namen von „Wissenschaft“ das Dogma einer Gruppe einer anderen aufgezwungen wird“" (Goldstein/Freud/Solnit/Goldstein1988: 115).

\section{Modernes Familien- und Familienverfahrensrecht}

Heutiges Familienrecht enthält Vor- und Leitbilder, regelt Rechtsverhältnisse, schützt erforderlichenfalls auch vor Familienmitgliedern, aber auch vor ungerechtfertigten Eingriffen des Staates, schlichtet, gleicht aus, versucht die Entscheidungsfähigkeit von Streitenden wiederherzustellen oder motiviert zur Annahme von Beratung, kennt aber auch Gebote zur Annahme von Hilfen bei möglicher Kindeswohlgefährdung. Das Familiengericht bezieht Kinder und Eltern oder sonstige Erziehungsberechtigte ein, kennt das Beratungsund Hilferepertoire freier und öffentlicher Träger der Kinder- und Jugendhilfe vor Ort und kann die Wirksamkeit angebotener und erbrachter Leistungen dieser Träger einschätzen, wozu auch die Einbeziehung der Hilfeplanung durch das Jugendamt nach dem $\S 36$ Abs. 2 Satz 2 SGB VIII (Sozialgesetzbuch, 8. Buch) auch in das familiengerichtliche Verfahren gehört (Schmid 2004: 86ff.). Zu diesem Idealbild eines modernen Familiengerichts gehört zudem, dass das kindliche Zeitempfinden durch Vorrang- und Beschleunigungsgebote Berücksichtigung findet. Da sich familiengerichtliche Tätigkeit ,auf die Lebenssitua- 
tion junger Menschen und ihrer Familien“ (§ 81 Nr. 2 SGB VIII) auswirkt, sind die Träger der öffentlichen Jugendhilfe gehalten, mit diesem zusammenzuarbeiten (§ 50 SGB VIII); zudem informiert das Jugendamt das Familiengericht insbesondere über angebotene und erbrachte Leistungen, bringt erzieherische und soziale Gesichtspunkte zur Entwicklung des Kindes oder des Jugendlichen ein und weist auf weitere Möglichkeiten der Hilfe hin (ibd.). Die Familiengerichte müssen auch in die Netzwerkstrukturen im Bereich Frühe Hilfen einbezogen werden ( 33 Abs. 2 KKG (Gesetz zur Kooperation und Information im Kinderschutz)).

So verwundert es kaum, dass Gesetzgeber im 20. Jahrhundert in zahlreichen Ländern mit zunehmender Tendenz familienrechtliche Entscheidungen speziellen Spruchkörpern innerhalb der Zivilgerichtbarkeit, nämlich Familiengerichten, zuweisen oder Zivilgerichte bei Familiensachen zur Beachtung besonderer Verfahrensregelungen verpflichten (s. Nakamura 1984). Dies ist die Konsequenz aus der Erkenntnis, dass in Familiensachen, insbesondere in Kindschaftssachen klassische Ausgleichs- und Regelungsmechanismen zivilrechtlicher, insbesondere vermögensrechtlicher Konflikte versagen (Salgo 1984: 225). Von Entscheidungen zum Sorge- und Umgangsrecht sind immer Kinder betroffen, auch wenn „nur“ Eltern untereinander oder Eltern mit der Kinder- und Jugendhilfe im Streit liegen; Interessenkonflikte, Machtgefälle und Disparitäten sind kennzeichnend; stets mit großen Unsicherheiten behaftete Prognosen spielen wie in kaum einem anderen Gebiet des Zivilrechts fast ständig eine wichtige Rolle. Materielles Familien-/Kindschaftsrecht ist hier nicht nur mit dem Verfahren in Familiensachen (FamFG), sondern auch mit der Kinder- und Jugendhilfe (SGB VIII) eng verzahnt: das Familiengericht (FamG) muss die Mitwirkung des Jugendamtes sicherstellen, muss prüfen, ob der Trennung des Kindes von der elterlichen Familie bei dessen Gefährdung durch öffentliche ambulante Hilfen vermieden werden kann ( $§ 1666$ a Abs. 1 Satz 1 BGB), Eltern zur Annahme von Beratung oder bei Gefährdung zur Annahme von öffentlichen Hilfen der Jugendhilfe zu motivieren sind, gegebenenfalls wird es Gebote zur Annahme von Leistungen der Kinder- und Jugendhilfe aussprechen, deren Nichteinhaltung weitere invasivere gerichtliche Eingriffe zur Folge haben kann. Jugendamt und Familiengericht sind gegenseitig aufeinander angewiesen (Interdependenz) (hierzu Sommer 2012).

\section{Entwicklungslinien des Familienrechts - ein Blick zurück und nach vorn}

Namhafte Autoren haben im 20. Jahrhundert kennzeichnende Beschreibungen zur Entwicklung des Familienrechts geliefert, die auch für die heutige Situation zutreffen:

- „Familienrecht ist in heißem Streit geworden“ (Martin Wolff, 1931)

- Musterbeispiel eines „sozial-nervösen und sensiblen Rechts“ (Rudolf Wiethölter, 1981)

- Ein mühseliger, immer wieder angezweifelter Kompromiss (Spiros Simitis, 1986)

Während die traditionell starken kirchlichen Bezüge und Einflussnahmen an Bedeutung zu verlieren scheinen, erfahren Fragen der Religion auch im säkularisierten Familienrecht einen Bedeutungszuwachs (z.B. religiöse Kindererziehung (Salgo 2006, 2014), Beschnei- 
dung (Salgo/Zenz/Fegert 2014; Staudinger/Salgo 2014: Erl. zu § 1631d BGB)). Gewissermaßen tritt an die Stelle kirchlichen Einflusses auf das Familienrecht dessen Konstitutionalisierung (Simitis 1994: 401ff.): vom Verfassungsrecht unbeeinflusste Nischen lassen sich hier kaum noch finden. Während in den 1980er und 1990er Jahren des vergangenen Jahrhunderts die Liste der „unerledigten Aufträge“ des Bundesverfassungsgericht an den Gesetzgeber (Salgo 1994) und der entsprechenden Monita des Europäischer Gerichtshof für Menschenrechte (EGHMR) im Bereich des Kindschaftsrechts immer länger wurde, ist inzwischen doch etliches rechtspolitisch aufgearbeitet worden, dennoch stellen Paarbeziehungen mit Kindern, Alleinerziehende und soziale Eltern in zunehmend mehr Varianten, das Abstammungsrecht, die Fortpflanzungstechnologien und Fragen des Kindeswohls in diversen Kontexten Familienrechtswissenschaft, Rechtsprechung und Gesetzgebung vor immer neue Herausforderungen - ohne human- und sozialwissenschaftliche Rückversicherung werden Wissenschaft, Gesetzgebung und Praxis sich diesen kaum stellen können.

Simitis (1994) beschrieb bereits vor über 20 Jahren diese Entwicklungslinien des Familienrechts zutreffend als:

- Universalisierung

- Verrechtlichung

- Prozeduralisierung

- Spezialisierung

- Bürokratisierung

- Verwissenschaftlichung

- Reprivatisierung

- Konstitutionalisierung.

Familien-/Kindschaftsrecht verdrängen als Sonderregelung zahlreiche Regelungen aus anderen Büchern des BGB (Koch 2013). Der leidenschaftliche Streit um Zugehörigkeit des Familienrechts zum öffentlichen oder zum Privatrecht (Müller-Freienfels 1973, 1974) wurde durch ein „Sowohl-als-auch“ bzw. durch ein ,aliud“ befriedet: beide Zuordnungen haben gute Argumente für und gegen sich; der Streit ist müßig, es gilt wie auch in anderen Bereichen sich mit hybriden Strukturen zu arrangieren. Die Fragen nach der Rolle des Staates bei der Regelung privater Beziehungen und der Schutz des Schwächeren sind dennoch aktueller denn je: „Ehe für alle“, „Scheidung ohne Richter“, „Regenbogenfamilien“, ,soziale Elternschaft“ (Scheiwe 2015), „nichteheliche Lebensgemeinschaft“, „Hochstrittigkeit“, Unterhalt zwischen Vergesellschaftung und Reprivatisierung, „Kinderschutz“ im Kontext von Sorge- und Umgangsrecht u.v.a.m.

\section{Familiengerichte und andere professionelle Akteure in Kindschaftssachen}

Zunächst war die Familiengerichtsbewegung (Salgo 1984) in Deutschland auf die Konzentration von zuvor bei verschiedenen Gerichten angesiedelten Angelegenheiten bei einem Gericht, nämlich dem neu geschaffenen Familiengericht als Abteilung des Amtsge- 
richts fixiert (1977-2009). Den sicherlich nur vorläufigen Abschluss fand diese Entwicklung mit einem ,großen Familiengericht“ durch das FGG-Reformgesetz (FGG-RG) (2009). Immer mehr stellt sich die Frage, ob die professionellen Akteure in Kindschaftssachen den hohen Anforderungen in diesem sensiblen und hochkomplexen Arbeitsfeld gerecht werden. Sind

- Familienrichter(innen),

- Verfahrensbeistände,

- Gutachter(innen),

- Rechtsanwältinnen/Rechtsanwälte,

- Mitarbeiter(innen) der Jugendämter

genügend für diese Verfahren qualifiziert?

\subsection{Qualifikation für richterliche Tätigkeit am Familiengericht}

Während die Eherechtskommission (1970/71) beim Bundesjustizministerium nicht nur die Zuständigkeitskonzentration beim Familiengericht gefordert hatte, war diese „ferner der Auffassung, dass in Ehesachen auch Erkenntnisse aus dem Bereich nichtjuristischer Disziplinen (z.B. Psychologie, Medizin, Sozialwissenschaften) zur Geltung kommen und erforderlichenfalls durch sachkundige Persönlichkeiten in das Verfahren eingeführt werden müssen“ (Eherechtskommission). Müller-Freienfels als Mitglied dieser Kommission hatte sich eindeutig positioniert: ,Je mehr soziologische, psychologische, pädagogische Kenntnisse der Richter besitzt und dabei mitverwertet, umso besser ist es" (Müller-Freienfels 1973: 127). Letztendlich konnte er sich aber - wie die regierungsamtliche Begründung zum 1. EheRG zeigt - nicht durchsetzen: „Eine besondere Vorbildung des Familienrichters, wie eine psychologische Schulung wird [neben hinreichender richterlicher Erfahrung] nicht verlangt; es kann erwartet werden, dass die Familiengerichte auch ohne ausdrückliche Regelung mit insoweit geeigneten Persönlichkeiten besetzt werden“ BT-Drucks. 7/650: 82). Dieser Standpunkt aus dem Jahre 1976 wird letztendlich bis heute im Bundesministerium der Justiz und für Verbraucherschutz (BMJV) vertreten. Mit der Einführung der Kindesanhörung durch das Sorgerechtsgesetz (1979) schien Bewegung in diese Kontroverse um die zusätzlich erforderliche Qualifikation für familienrichterliche Tätigkeit zu kommen, zumal das BVerfG den nachfolgenden Ansatz des Rechts- und Familienausschusses bekräftigte: die ,an Familien- und Vormundschaftsgerichten tätigen Richter (sollten) durch Aus- und Weiterbildung mit den Grundzügen der Pädagogik und Psychologie vertraut gemacht und dadurch befähigt werden (...), in größerem Umfang als bisher die betroffenen Kinder und Jugendlichen selbst zu hören“" (BT-Drucks. 8/2788. 42; BVerfG 55, 171,180).

Während zunächst eine dreijährige richterliche Erfahrung Voraussetzung für eine Tätigkeit am Familiengericht war, wurde diese im Zuge der deutschen Wiedervereinigung auf ein Jahr reduziert ( $\$ 23$ Abs. 3, S. 3 GVG (Gerichtsverfassungsgesetz)) - weitere Voraussetzungen bestehen bis heute nicht, obschon - wie gezeigt werden konnte - die Anforderungen enorm gewachsen sind. Es geht hier nicht nur um Qualifizierung in außerrechtlichen Bereichen, sondern oft auch um fehlende Kenntnisse in der Richterschaft im Bereich des Kinder- und Jugendhilferechts (SGB VIII), dessen Bezugspunkte in Kind- 
schaftssachen offensichtlich sind. Seit Familiengerichte bestehen, gibt es eine anhaltende Diskussion um zusätzliche Qualifikationserfordernisse und insbesondere um die Fortbildungspflicht, deren Bestehen grundsätzlich nicht in Frage gestellt wird, deren Wahrnehmung aber jeder Familienrichterin und jedem Familienrichter selbst überlassen bleibt (Bundesministerium der Justiz und für Verbraucherschutz) und nicht durch Anreize und entsprechende überprüfbare Verpflichtungen sichergestellt wird. Nicht nur, dass es für Familienrichter und Familienrichterinnen keine Entlastung bei Teilnahme an Fortbildung gibt, selbst über die Relevanz von Fortbildung bei Beförderungen oder bei der Übernahme der Reisekosten besteht eine uneinheitliche Praxis (Arbeitsgruppe „Familiengerichtliche Maßnahmen bei Gefährdung des Kindeswohls - § 1666 BGB“ 2009: 26).

7.1.1. Der neuere Stand der Diskussion zur Fortbildungspflicht für an Familiengerichten tätige Richter(innen)

Es verwundert schon, dass sich bislang die Rechtspolitik auf Bundes- wie Länderebene nicht zu einer Fortbildungspflicht für Richter und Richterinnen mit einer entsprechenden Nachweispflicht durchringen konnte, obwohl sich die Konferenz der Justizministerinnen und Justizminister (JuMiKo) schon im Jahre 2005 für eine Verankerung der Fortbildungspflicht in den Richtergesetzen des Bundes und der Länder ausgesprochen hat. Lehrerinnen und Lehrer, Ärztinnen und Ärzte, aber auch z.B. Fachanwältinnen und Fachanwälte, demnächst wahrscheinlich alle Rechtsanwältinnen und Rechtsanwälte, müssen regelmäßig nachweisen, dass sie an Fortbildungen mit einer Mindeststundenzahl bei dafür anerkannten Trägern teilgenommen haben ( $\$ 15$ Fachanwaltsordnung: ,anwaltsorientierte oder interdisziplinäre Veranstaltung"). Überzeugende Argumente dafür, dass entsprechende Verpflichtungen für Richterinnen und Richter nicht erforderlich sind, konnten bis heute nicht vorgetragen werden.

Das BMJV vertritt nachfolgenden Standpunkt:

„Eine weitere Qualitätssicherung richterlicher Tätigkeit im Sinne einer Überprüfung des Richters, die über die verfahrensrechtliche Möglichkeit der Überprüfung einer Entscheidung durch Einlegung eines Rechtsmittels einerseits und über den Weg der Dienstaufsicht hinausginge, wäre wegen Verstoßes gegen die richterliche Unabhängigkeit gemäß Artikel 97 des Grundgesetzes (GG) unzulässig“" (Bundesministerium der Justiz und für Verbraucherschutz).

Diese Auffassung befremdet: Die gegenteilige Auffassung dürfte richtig sein. Nicht eine Fortbildungspflicht gefährdet die richterliche Unabhängigkeit, sondern der Umstand, dass das für die Tätigkeit in Kindschaftssachen erforderliche Wissen häufig nicht vorhanden ist bzw. nicht aufgefrischt werden muss. Nun darf nicht verschwiegen werden, dass es durchaus vereinzelte und beachtliche Aktivitäten in den Bundesländern gibt, um diese Wissenslücken aufzufüllen. Was es bundesweit nicht gibt, ist eine entsprechende einheitliche curriculare Orientierung und eine Teilnahmepflicht. Es gibt zahlreiche Einführungsveranstaltungen in die Tätigkeit am Familiengericht, allerdings finden die kindschaftsrechtlichen Themen neben den vielen anderen wichtigen kaum Platz, das gilt erst recht für die nichtrechtlichen Themen (vgl. ibd.: 26f.).

Letztendlich stellt sich die Frage, welche Justiz wir uns leisten wollen und können. Fehlentscheidungen der Justiz sind sehr teuer, individuell und für die Gesellschaft. Es fällt auf, dass die breite Fachdiskussion um Fehler im beruflichen Handeln von Fachkräften in 
der Kinder- und Jugendhilfe („Fehlerkultur") oder auch in der Medizin überhaupt keine Entsprechung bei den Familiengerichten findet, obwohl etwa in den bekannt gewordenen Todesfällen von Kindern immer wieder auch Familiengerichte, aber auch Staatsanwaltschaften noch sogar zu Lebzeiten der Kinder involviert waren. Strukturelle Defizite werden ja bekanntlich nicht durch die rechtsförmigen Rechtsbehelfe, auf die das BMJV verweist, aufgearbeitet. Die Kostenfrage wird kaum einmal offen angesprochen: es geht um die Fortbildung von ca. 20.000 Richter(inne)n in den Bundesländern und von ca. 500 Bundesrichter(inne)n. Das bestehende Angebot an freiwilliger Fortbildung dürfte bei weitem nicht den Bedarf abdecken. Angesichts der seit Jahren ablehnenden Haltung der Justizpolitik in Bund und Ländern müssten die Richter und Staatsanwälte selbst die Initiative ergreifen: um ihre herausfordernden Aufgaben zu bewältigen, sollten sie einen Rechtsanspruch auf regelmäßige hochqualifizierte Fortbildung haben, die Teilnahme hieran sollte auch nachgewiesen werden müssen und beförderungsrelevant sein. Angesichts der ohnehin bestehenden Überlastung der Justiz muss dieser Nachweis auch Auswirkungen auf die Arbeitsbelastung (auf Pensenschlüssel) haben. Falls die Richterschaft selbst einen Rechtsanspruch gekoppelt mit einer Fortbildungspflicht fordern würde, wäre es für die Länder und den Bund nicht so einfach, dies abzulehnen.

Wie viele der Familienrichter(innen) von sich aus an freiwilligen Fortbildungsangeboten teilnehmen, wird aus vorliegenden Umfragen nicht deutlich. Es wird zwar von regem Interesse an solchen Angeboten berichtet, zugleich aus einem Bundesland mitgeteilt, „dass es eine gleichbleibende Gruppe fortbildungsinteressierter Richterinnen und Richter gebe, während die Vielzahl der Richterinnen und Richter sich selten oder nie zu Fortbildungen anmelde" (ibd.: 26).

\subsection{2. „Weiche“ Themen in Fortbildungsveranstaltungen}

Vorherrschend finden sich in den Fortbildungsangeboten rechtliche (also „harte“) Themen, wie Unterhalt, Versorgungs- und Zugewinnausgleich, FGG-RG. Aber auch „weiche" Themen erlangen zunehmend an Gewicht (z.B. interdisziplinäre Kooperation, Vernetzung, Kommunikation mit dem Kind).

Obwohl Vorschläge einer vom BMJ eingesetzten Arbeitsgruppe aus dem Jahre 2006 keinerlei Umsetzung erfuhren und im BMJV inzwischen der Vergessenheit anheimgefallen zu sein scheinen, bleiben diese aktuell:

- „Die Arbeitsgruppe schlägt den Ländern und dem Bund vor, eine allgemeine Fortbildungspflicht für Richter ausdrücklich gesetzlich $\mathrm{zu}$ verankern („Richterinnen und Richter sind verpflichtet, sich fortzubilden.").

- Die nötigen finanziellen und sachlichen Mittel für Fortbildungen sollten bereitgestellt und die für Fortbildungen nötigen zeitlichen Kapazitäten bei den Richterinnen und Richtern ermöglicht werden.

- Es sollten Konzepte entwickelt werden, wie auch diejenigen Richterinnen und Richter erreicht werden können, die bislang selten an Fortbildungen teilnehmen.

- Die Kommission der Landesjustizverwaltungen für Fragen der Personalbedarfsberechnung sollte nochmals eine Anpassung von PEPP\$Y (Personalbedarfsberechnungssystem) im Hinblick auf die Teilnahme von Richterinnen und Richtern an interdisziplinären Arbeitskreisen überprüfen“ (ibd.: 27, 31). 
Als Probleme der Praxis nennt die Arbeitsgruppe u.a.: „Umgangsrecht und Sorgerecht mit psychologischen Aspekten, Jugendhilferecht, verfahrensrechtliche Besonderheiten im familiengerichtlichen Verfahren“, sie ist der Ansicht, dass Familienrichter und -richterinnen

„gerade in Umgangs- und Sorgerechtssachen über kinderpsychologische und sozialpädagogische Grundkenntnisse sowie über Gesprächsführungskompetenzen in konflikthaften Familiensituationen verfügen“ sollten; sie legt Wert darauf, dass diese „Kenntnisse (...) laufend auf dem neuesten Stand gehalten werden" sollten (ibd.: 26).

Bemühungen der damaligen Justizministerin scheiterten bedauerlicherweise, wohl an den Finanzministern der Länder. So verwundert es kaum, dass auch der amtierende Minister der Justiz und für Verbraucherschutz sich nicht erneut Absagen von Seiten der Bundesländer einholen will. Beschlüsse und Prüfbitten der Justizministerkonferenz bleiben oft ohne Erfolg. In der Anhörung zum Sachverständigenrecht im Rechtsausschuss des Deutschen Bundestages bekam die Richterfortbildung, obwohl sie nicht Beratungsgegenstand war (es ging um die Qualität von Sachverständigengutachten) jüngst zuvor nicht gekannte Aufmerksamkeit der Abgeordneten (BT-Drucks. 18/6985; Protokoll Nr. 18/94, Deutscher Bundestag, Ausschuss für Recht und Verbraucherschutz). Der Deutsche Richterbund lehnt in einem, im übrigen bemerkenswerten Eckpunktepapier vom 29.04.2016 nach wie vor eine Fortbildungspflicht ab.

In einen Katalog der „weichen“ Themen der Fortbildung für familienrichterliche Tätigkeit im Kindschaftsrecht insbesondere gehören:

- Methoden der Kindesanhörung

- Auswahl geeigneter Gutachter und die richtige Fragestellung an diesen

- Umgang mit jugendamtlichen Stellungnahmen

- Auswirkungen von häuslicher Gewalt, auch des Miterlebens, auf Kinder

- Folgen von Traumatisierungen in unterschiedlichen Altersstufen

- Grundlagen der Entwicklungspsychologie

- Bedeutung von Kontinuität, Bindung (hierzu Fegert/Kliemann 2014: 173ff.) und Trennung im Kindesalter

- Bedeutung des kindlichen Zeitempfindens

- Wirkung von Hochstrittigkeit auf Kinder

- Erzwungener Umgang

- Möglichkeiten und Grenzen von „Hilfen zur Erziehung“, Beratung, Mediation und deren (Aus-)Wirkungen auf Kinder

- Wirkungen von Zwangskontexten und Geboten

- Kindeswille und Kindeswohl.

In schwierige Gelände des Familienrechts scheint sich das BMJV ohnehin nicht freiwillig zu begeben, wie sich auch an der Absage an die Prüfbitte der 84. Justizministerkonferenz (12. Juni 2013) ersehen lässt; diese hatte das BMJ gebeten, zu untersuchen, „ob und ggf. wie durch gesetzliche Regelungen die rechtliche Position von Pflegefamilien in lang dauernden Pflegeverhältnissen im Interesse des Kindeswohls verbessert werden kann“. Das BMJV kam zunächst zu dem Ergebnis, ,dass eine gesetzliche Regelung im Kindschaftsrecht derzeit nicht angezeigt, aber auch nicht erforderlich ist" (Schreiben des BMJV v. 12. 05.2015 an die JuMiKo). Inzwischen sieht auch der Bundesminister für Justiz und Ver- 
braucherschutz (s. Katholische Nachrichtenagentur (KNA) vom 26.08.2015), wie auch die Bundesregierung, Handlungsbedarf (BT-Drucks. 7100, S. 4, 77; s. auch DionaniStreek/Salgo 2016) Zahlreiche Experten und Verbände (Deutscher Familiengerichtstag, Kinderrechtekommission) haben sich hierzu geäußert (Wissenschaftlicher Beirat für Familienfragen 2016).

\subsection{Qualität in der Fachanwaltschaft}

Grundsätzlich scheint sich die Möglichkeit, Fachanwaltsbezeichnungen - wie z.B. Fachanwalt/Fachanwältin für Familienrecht - erlangen zu können, bewährt zu haben. Allerdings haben in den entsprechenden Curricula, auch wenn diese in $\S 12$ Nr. 1 Fachanwaltsordnung AO benannt sind, kindschaftsrechtliche Themen eine eher untergeordnete Bedeutung; das wichtige SGB VIII taucht hier überhaupt nicht auf. Soweit Fachanwälte verstärkt im kindschaftsrechtlichen Bereich tätig sein wollten, so kann hier auf die bereits benannten wichtigen Inhalte einer Fortbildung zu „weichen“ Themen für die Familiengerichte verwiesen werden. Auch und gerade die benannten „weichen“ Themen wären ebenso für eine rechtsanwaltliche Tätigkeit im Bereich des Kindschaftsrechts wichtig. Das eher vorzufindende Desinteresse an kindschaftsrechtlichen Themen in der deutschen Anwaltschaft mag mit den Herausforderungen zeitlicher Art wie auch den psychischen Belastungen, vor allem aber mit den Regelstreitwerten zusammenhängen; solange Fachanwälte i.d.R. nicht auch ausgewiesene Kenner des Kindschaftsrechts, aber auch der dazugehörigen „weichen Themen“ sind, wird eine Erhöhung des Regelstreitwerts kaum gefordert werden können.

\subsection{Qualität in der Verfahrensbeistandschaft}

Immerhin darf das Familiengericht inzwischen gemäß $§ 158$ Abs. 1 FamFG nur „einen geeigneten Verfahrensbeistand bestellen“. Wie das Familiengericht die Eignung überprüft, bleibt ihm überlassen. Inzwischen haben Fortbildungsträger recht anspruchsvolle Fortbildungskurse entwickelt, an denen eher Teilnehmer/innen mit einer Grundausbildung in Sozialarbeit/-pädagogik, Psychologie, zunehmend aber auch Rechtsanwältinnen und Rechtsanwälte teilnehmen.

Verfahrensbeistände sollten (Salgo/Zenz/Fegert 2014: 17):

- über solide Rechtskenntnisse auf dem Gebiet des Familien-, insbesondere des Kindschaftsrechts verfügen,

- die Komplexität des für Kinder und Jugendliche und ihre Eltern relevanten Familienverfahrensrechts beherrschen,

- gründliche Kenntnisse zum SGB VIII haben,

- Kenntnisse der sozialen und psychischen Lebenssituation von Minderjährigen, die auf eigenständige Interessenvertretung angewiesen sind, haben (solche Situationen sind insbesondere: streitiges Umgangs- und/oder Sorgerecht, häusliche Gewalt, Misshandlung, Vernachlässigung, sexuelle Ausbeutung, Fremdplazierung, Adoption, freiheitsentziehende Unterbringung rechtfertigende Ausgangslagen), 
- über Grundkenntnisse in Entwicklungspsychologie verfügen,

- Techniken kennen und über Kompetenzen verfügen, um Minderjährige zu verstehen und um mit ihnen zu kommunizieren,

- über Vermittlungskompetenzen verfügen und

- Kenntnisse der Angebote öffentlicher und freier Träger der Kinder- und Jugendhilfe vor Ort haben.

Familienrichter/innen scheinen zunehmend Verfahrensbeistände mit diesen Zusatzqualifikationen zu schätzen (Rabe 2007: 437) und beizuordnen, weil deren Kenntnisse für die Verfahren, in denen Verfahrensbeistände zu bestellen sind, sehr hilfreich sind. Eine human-wissenschaftliche Grundorientierung gepaart mit einschlägigen Rechtskenntnissen erleichtert die Auswahlentscheidung des Familiengerichts.

\subsection{Qualität der Sachverständigengutachten}

Die Bundesregierung hat jüngst einen Entwurf eines „Gesetzes zur Änderung des Sachverständigenrechts und zur weiteren Änderung des Gesetzes über das Verfahren in Familiensachen und in den Angelegenheiten der freiwilligen Gerichtsbarkeit" (BT-Drucks. 18/6985; s. auch: Mindestanforderungen an die Qualität von Sachverständigengutachten im Kindschaftsrecht, Arbeitsgruppe Familienrechtliche Gutachter 2015) vorgelegt. Ziel ist die Verbesserung der Qualität von Gutachten sowie die Gewährleistung der Neutralität gerichtlich beigezogener Sachverständiger. Im Zentrum dieses Entwurfs steht dessen $\S$ 163 Abs. 1 FamFG-E(ntwurf):

„In Verfahren nach $\S 151$ Nummer 1 bis 3 ist das Gutachten durch einen geeigneten Sachverständigen zu erstatten, der mindestens über eine psychologische, psychotherapeutische, kinder- und jugendpsychiatrische, psychiatrische, ärztliche, pädagogische oder sozialpädagogische Berufsqualifikation verfügen soll.“

Grundsätzlich könnte dieser Vorschlag Verbesserungen bringen; jedoch zeigt sich auch hier, dass nur ein(e) zusätzlich fortgebildete/r Familienrichter(in) in der Lage sein wird die Auswahlentscheidung verantwortlich zu treffen, zu begründen und das Gutachten inhaltlich würdigen zu können.

\subsection{Qualität der jugendamtlichen Mitwirkung}

Auch hier bestehen erhebliche Verbesserungsbedarfe; teilweise weniger zu den genannten „weichen“ Themen. Allerdings sind auch in den modularisierten BA-Studiengängen die Kommunikation mit Kindern, das Kindschafts- und Jugendhilferecht, insbesondere zivilrechtlicher und behördlicher Kinderschutz und das FamFG keineswegs an allen Hochschulen und Universitäten in den einschlägigen Studiengängen verbindlicher Unterrichtsgegenstand. Bemerkenswert ist die Sicherstellung des Verstehens und der Anwendung von Recht im Jugendamt München: hier beraten und unterstützen hochspezialisierte Juristen und Juristinnen, die im Jugendamt eingegliedert sind, die Fachkräfte der Kinder- und Jugendhilfe zu allen relevanten Rechtsfragen dieses Tätigkeitsfeld. 


\section{Resümee}

Die Beziehung zwischen Kindschaftsrecht und den Human-/Sozialwissenschaften ist so offensichtlich, dass eine Klärung und Systematisierung dieses Verhältnisses überflüssig schien. Der Ruf nach einer Integration der Human- und Sozialwissenschaften in Rechtswissenschaft und in Rechtsanwendung war in den 1970er und 1980er Jahren des vergangenen Jahrhunderts en vogue; sie wurde in Ansätzen verwirklicht, aber auch wieder zurückgenommen. Die Notwendigkeit, Erkenntnisse der Human-/Sozialwissenschaften in der Familienrechtswissenschaft, der Gesetzgebung und der Rechtspraxis zu beachten, ist aktueller denn je. In der Abhandlung konnten lediglich die Berührungsbereiche am Beispiel des Kindschaftsrechts aufgezeigt werden. Für das Kindschaftsrecht besteht die berechtigte Hoffnung, dass Gesetzgebung und Rechtsanwendung unter Heranziehung und Einbeziehung human-/sozialwissenschaftlicher Methoden und Wissensbestände am ehesten eine dem Wohl des Kindes am besten gerecht werdende - besser: eine dem Wohl des Kindes am wenigsten schädliche - Alternative finden.

\section{Literatur}

Arbeitsgruppe „Familiengerichtliche Maßnahmen bei Gefährdung des Kindeswohls - § 1666 BGB“ (2009). Abschlussbericht vom 14. Juli 2009.

Berneiser, C. (2015). Die verfahrensrechtliche Neuregelung der Erörterung der Kindeswohlgefährdung in $\$ 157$ FamFG. Frankfurt am Main: Peter Lang (Studien zum deutschen und internationalen Familien- und Erbrecht - Band 26).

Coester, M. (1983). Das Kindeswohl als Rechtsbegriff, Das Kindeswohl als Rechtsbegriff. Die richterliche Entscheidung über die elterliche Sorge beim Zerfall der Familiengemeinschaft. Frankfurt am Main: Metzner (Arbeiten zur Rechtsvergleichung, Band 114), S. 178, Fußnote 13, mit weiteren Nachweisen.

Deutscher Familiengerichtstag, Kinderrechtekommission (2014). Reformbedarf im Pflegekinderwesen. Zeitschrift für das gesamte Familienrecht (FamRZ), 2014, 11, S. 891-902.

Dionani-Streek, M.(Salgo, L. (2016). Probleme sozialer Elternschaft für Pflegeeltern und Vorschläge zu ihrer rechtlichen Anerkennung. Recht der Jugend und des Bildungswesens (RdJB), 2016, 4, S. 176193.

Eherechtskommission (1971). Bericht der Eherechtskommission beim Bundesministerium der Justiz zur Eherechtsreform, II. Teilbericht: Vorschläge zur Reform des Verfahrensrechts in Ehesachen. Bielefeld: Gieseking.

Enneccerus L, Kipp, T. \& Wolf, M. (1931). Lehrbuch des bürgerlichen Rechts, Band 4. Das Familienrecht. Marburg: Elwert'sche Verlagsbuchhandlung (7. Auflage).

Fegert, J. M. (2013). Endgültiges Aus für das Parental Alienation Syndrome (PAS) im amerikanischen Klassifikationssystem DSM-5. Zeitschrift für Kindschaftsrecht und Jugendhilfe (ZJK), 2013, 5, S. 190-191 (mit weiteren Nachweisen).

Fegert, M./Kliemann, A. (2014). Das Verständnis von Bindung in Entwicklungspsychologie, Entwicklungspathologie und Familienrecht. Zirkelschlüsse und Missverständnisse. In: Götz, I., Schwenzer, L., Seelmann, K. \& Taupitz, J. (Hrsg.), Familie - Recht - Ethik. Festschrift für Gerd Brudermüller zum 65. Geburtstag. München: C. H. Beck, S. 173-188.

Goldstein J., Freud, A., Solnit, A. \& Goldstein, S. (1988). Das Wohl des Kindes. Grenzen professionellen Handelns. Frankfurt am Main: suhrkamp taschenbuch wissenschaft.

Hartwig, O. \& Rebe, B. (1978). Familienrecht und Familiensoziologie. In: Kühn E. \& Tourneau, I. (Hrsg.), Familienrechtsreform - Chancen einer besseren Wirklichkeit. Bielefeld: Gieseking, S.17, 24ff. 
Justizministerkonferenz. 76. Konferenz vom 29. bis 30. Juni 2005 in Dortmund.

Kindler, H. (2013). Trennungen zwischen Kindern und Bezugspersonen. Familie, Partnerschaft, Recht (FPR), 2013, 5, S. 194-199.

Koalitionsvertrag zwischen CDU, CSU und SPD. 18. Legislaturperiode.

Koch, E. (2013). Dogmatische Besonderheiten des Familienrechts In: Münchener Kommentar: Einleitung vor $\$ 1297$. München: C. H. Beck, Randnotiz 46ff.

Kostka, K. (2014). Das Wechselmodell als Leitbild? Umgang und Kindeswohl im Spiegel aktueller internationaler Forschung. STREIT. Feministische Rechtzeitschrift, 2014, 4, S. 147.

Luhmann, N. (1983). Legitimation durch Verfahren. Frankfurt am Main: suhrkamp taschenbuch wissenschaft.

Müller-Freienfels, W. (1973). Über Familiengerichte, insbesondere in den USA (zugleich Besprechung von Otto von Busekist, Das Familiengericht in der vereinigten Staaten von Amerika. Ein Beitrag zur Reform des Ehescheidungsverfahrens, 1970). Zeitschrift für Vergleichende Rechtswissenschaft (ZVglRWiss), 73, S. 118.

Müller-Freienfels, W. (1973, 1974). Die Diskussion um die systematische Einordnung des Familienrechts, Rabels Zeitschrift für ausländisches und internationales Privatrecht (RabelsZ), 37/38, S. 609 (1973), 533 (1974).

Nakamura, H. (Hrsg.) (1984). Familiengerichtsbarkeit. Nationalberichte zum 7. Internationalen Kongreß für Prozeßrecht. Tokio.

Rabe, H. (2007). Die Verfahrenspflegschaft zwischen fortschreitender Umsetzung und Novellierung. Zeitschrift für Kindschaftsrecht und Jugendhilfe (ZKJ) 2007, 11, S. 437-444.

Röthel, A. (2015). Arbeitsweisen der Familienrechtswissenschaft. In: Hilbig-Lugani, K., Jacob, D., Mäsch, G. et al. (Hrsg.), Zwischenbilanz, Festschrift für Dagmar Coester-Waltjen zum 70. Geburtstag. Bielefeld: Gieseking, S.183-192.

Salgo, L. (1984). Soll die Zuständigkeit des Familiengerichts erweitert werden? Zeitschrift für das gesamte Familienrecht (FamRZ), 1984, S. 221, 225

Salgo, L. (1994). Unerledigte „Aufträge“ des Bundesverfassungsgerichts an den Gesetzgeber auf dem Gebiet des Familienrechts. Kritische Vierteljahresschrift für Gesetzgebung und Rechtswissenschaft (KritV), 1994, S. 262

Salgo, L. (2006). Religiöse Kindererziehung (k)ein Thema für das Recht im 21. Jahrhundert?! In: AdolfArndt-Kreis (Hrsg.), Nun sag, wie hast Du's mit der Religion?. Berlin: Berliner WissenschaftsVerlag, S. 45-55.

Salgo, L. (2009). „Ein Schritt nach vorn, zwei Schritte zurück“ - Kritische Anmerkungen zur Installierung des Umgangspflegers und zur Revision der Verfahrenspflegschaft im FGG-RG, in: Lipp, V., Schumann, E. \& Veit, B. (Hrsg.), Reform des familiengerichtlichen Verfahrens. 1. Familiengerichtliches Forum Göttingen. Göttingen: Universitätsverlag Göttingen, S.153-194.

Salgo, L., Zenz, G. \& Fegert, J. M. (Hrsg.) (2014). Verfahrensbeistandschaft. Ein Handbuch für die Praxis. Köln: Bundesanzeiger Verlag.

Scheiwe, K. (2015), Die Ausübung elterlicher Sorgerechte durch soziale Eltern. In: Hilbig-Lugani, K., Jacob, D., Mäsch, G. et al. (Hrsg.), Zwischenbilanz, Festschrift für Dagmar Coester-Waltjen zum 70. Geburtstag. Bielefeld: Gieseking, S. 205-222.

Schmid, H. (2004). Die Hilfeplanung nach § 36 SGB VIII. Frankfurt am Main: Eigenverlag des Deutschen Vereins für öffentliche und private Fürsorge.

Simitis, S. (1986). Kindschaftsrecht - Elemente einer Theorie des Familienrechts. In: Dieckmann A., Frank, R., Hanisch, H. \& Simitis, S. (Hrsg.), Festschrift für Wolfram Müller-Freienfels. BadenBaden: Nomos, S. 579-616.

Simitis, S. (1994). Familienrecht. In: Simon, D. (Hrsg.), Rechtswissenschaft in der Bonner Republik. Studien zur Wissenschaftsgeschichte der Jurisprudenz. Frankfurt am Main: Suhrkamp, S. 390, $425 \mathrm{ff}$.

Sommer, A. (2012). Das Verhältnis von Familiengericht und Jugendamt. Frankfurt am Main: Peter Lang. 
Wiethölter, R. \& Bernhardt, R. (1968). Rechtswissenschaft. Frankfurt am Main: Fischer (Funk-Kolleg zum Verständnis der modernen Gesellschaft, Band 4).

Wissenschaftlicher Beirat für Familienfragen (2016). Pflegefamilien als soziale Familien, ihre rechtliche Anerkennung und aktuelle Herausforderungen. Berlin: BMFSFJ.

Eingereicht am/Submitted on: 15.10.2015

Angenommen am/Accepted on: 08.04.2016

Anschrift des Autors/Address of the author:

Prof. Dr. Ludwig Salgo

Goethe-Universität

Fachbereich Rechtswissenschaft

Senckenberganlage 31

60325 Frankfurt am Main

Deutschland/Germany

E-Mail: Salgo@jur.uni-frankfurt.de 